\title{
Giant molecular clouds and star formation in the Large Magellanic Cloud
}

\author{
A. Kawamura ${ }^{1}$, T. Minamidani ${ }^{1}$, Y. Mizuno ${ }^{1}$, \\ T. Onishi ${ }^{1}$, N. Mizuno ${ }^{1}$, A. Mizuno ${ }^{2}$ and Y. Fukui ${ }^{1}$ \\ ${ }^{1}$ Department of Astrophysics, Nagoya University, Chikusa-ku, Nagoya 464-8602, Japan \\ email: kawamura@a.phys.nagoya-u.ac.jp \\ ${ }^{2}$ Solar-terrestrial Environment Lab., Nagoya University, Chikusa-ku, Nagoya 464-8601, Japan
}

\begin{abstract}
In order to elucidate star formation in the Large Magellanic Cloud, a complete survey of the molecular clouds was carried out by NANTEN. In this work, we compare 230 giant molecular clouds (GMCs), whose physical quantities are well determined, with young clusters and $\mathrm{H}$ II regions. We find that about $76 \%$ of the GMCs are actively forming stars or clusters, while $24 \%$ show no signs of massive star or cluster formation. Effects of supergiant shells (SGSs) on the formation of GMCs and stars are also studied. The number and surface mass densities of the GMCs are higher by a factor of 1.5-2 at the edge of the SGSs than elsewhere. It is also found that young stellar clusters are more actively formed in the GMCs facing to the center of the SGSs. These results are consistent with the previous studies by Yamaguchi et al. and suggest the formation of GMCs and the cluster is triggered by dynamical effects of the SGSs.
\end{abstract}

Keywords. stars: formation, ISM: clouds, Magellanic Clouds, galaxies: star clusters

\section{Introduction}

The Large Magellanic Cloud (LMC), at a distance of $\sim 50 \mathrm{kpc}$, is one of the nearest galaxies to our own. Studies of the LMC provide invaluable information to understand the evolution of interstellar matter (ISM) and star formation. The relatively face-on inclination of the LMC also enables us to obtain a sample of astronomical objects with little contamination.

Studies of the distribution and the properties of the young stars and clusters have been carried out by optical, infrared and radio continuum observations for decades. More than $300 \mathrm{H}$ II regions are identified (e.g., Davies et al. 1976). Stellar clusters called "populous clusters" with masses $\sim 10^{4}-10^{5} M_{\odot}$, were found by photometric studies (e.g., Hodge 1961). There are populous clusters significantly younger, i.e., a few Myr-10 Gyr, than the Galactic globular clusters and some populous clusters are still being formed at present (e.g., Brandl et al. 1996). Not only the star formation indicators but also the structure of the ISM has been studied, for example, in H $\alpha$ and H I (e.g., Meaburn 1980; Kim et al. 1999). One of the remarkable feature is the existence of various shells and supergiant shells (SGSs) as commonly found in nearby galaxies (e.g., Tenorio-Tagle \& Bodenheimer 1988). The SGSs in the LMC consist of long $\mathrm{H} \alpha$ filaments and bright $\mathrm{H}$ II regions, and have more than a few 10 to 100 OB stars in their interior. Nine SGSs were cataloged by Meaburn (1980). Since all of the nine $\mathrm{H} \alpha$ SGSs contain numerous OB stars inside the SGSs, they are likely to be formed due to the effects of stellar winds and supernovae. In this work, we compare the molecular clouds and young clusters with the nine $\mathrm{H} \alpha$ SGSs. 

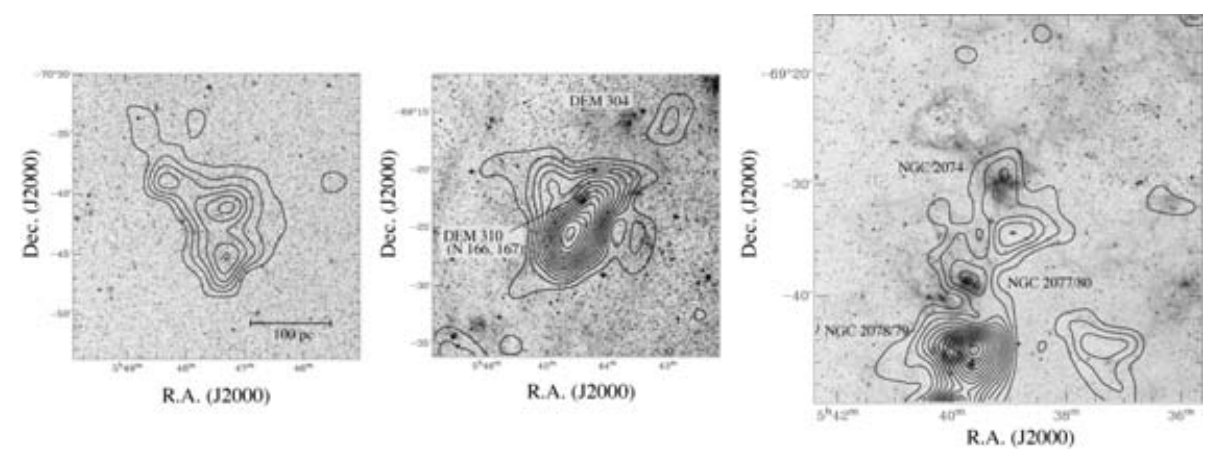

Figure 1. Examples of (Left) Class I GMC with no signs of massive star or cluster formation, (Middle) Class II GMC associated with H II regions, and (Right) Class III GMC associated with $\mathrm{H}$ II regions and clusters $(\tau<10 \mathrm{Myr})$. Images are from DSS2 and the contours are from the NANTEN survey. The contour levels are from $1.2 \mathrm{~K} \mathrm{~km} \mathrm{~s}^{-1}$ with $1.2 \mathrm{~K} \mathrm{~km} \mathrm{~s}^{-1}$ intervals.

\section{Giant molecular clouds}

We have carried out $\mathrm{CO}(1-0)$ observations toward the LMC with NANTEN, a $4 \mathrm{~m}$ telescope of Nagoya University at Las Campanas Observatory, Chile from 1998 April to 2003 August. The resolution is $\sim 40 \mathrm{pc}$ at a distance of the LMC. The $3 \sigma$ noise level of the velocity-integrated intensity is $\sim 1.2 \mathrm{~K} \mathrm{~km} \mathrm{~s}^{-1}$. This corresponds to $N\left(\mathrm{H}_{2}\right) \sim 8 \times$ $10^{20} \mathrm{~cm}^{-2}$, by assuming a conversion factor from $\mathrm{CO}$ intensity to the hydrogen column density, $X=7 \times 10^{20} \mathrm{~cm}^{-2}\left(\mathrm{~K} \mathrm{~km} \mathrm{~s}^{-1}\right)^{-1}$. We identified 272 molecular clouds by using fitstoprops method (Rosolowsky \& Leroy 2006), 230 out of which are detected at more than 2 observed positions (for the details, see Fukui et al. 2006).

\section{Massive star and cluster formation}

We made comparisons of the 230 GMCs with H II regions (e.g., Davies et al. 1976, Filipovic et al. 1998) and young clusters (e.g., Bica et al. 1996). Bica et al. (1996) studied the colors of the clusters and classified them into an age sequence from SWB0 to SWB VII. The datasets of $\mathrm{H}$ II regions have a detection limit in $\mathrm{H} \alpha$ flux of $\sim 10^{-12} \mathrm{ergs} \mathrm{cm}^{-2}$ $\mathrm{s}^{-1}$ (Kennicutt \& Hodge 1986). In order to examine any optically obscured H II regions we have also used the Parkes/ATNF radio continuum survey (Dickel et al. 2005; Filipovic et al. 2006). The typical sensitivity of the new datasets is good enough to reach the flux limit equivalent to that of $\mathrm{H} \alpha$. We note that the detection limit of $\mathrm{H}$ II regions is quite high, corresponding to one-fourth the luminosity of the Orion Nebula.

A study of the distance of $\mathrm{H}$ II regions and clusters measured from the nearest GMCs shows that a large number of the young clusters $(\tau<10 \mathrm{Myr})$ and $\mathrm{H}$ II regions are found within 100 pc of the GMCs. On the other hand, older clusters show almost no correlation with the GMCs. We have examined the association between the individual GMCs and the $\mathrm{H}$ II regions and young clusters and found that about a half of the $\mathrm{H}$ II regions and young clusters are associated with the GMCs. The GMCs are classified into three classes; Class I. starless GMCs (no early O stars), Class II. GMCs with H II regions only, and Class III. GMCs with H II regions and stellar clusters (Fig. 1, see also Fukui et al. 1999, Yamaguchi et al. 2001b). It is found that $\sim 24 \%$ of the GMCs are starless (Class I), while $52 \%$ (Class II) and 24\% (Class III) are associated with H II regions and young clusters, respectively. Figure 2 shows the mass distribution of the three GMC classes. It is shown that the number ratio of massive GMCs is higher in Class III than those in the other two, and the mass of Class I GMCs tends to be smaller than the rest. We may speculate 
that Class I, and possibly Class II GMCs, are still growing in mass via mass accretion from their surrounding lower density atomic gas (Fukui 2006 in this proceedings).

The completeness of the present GMC

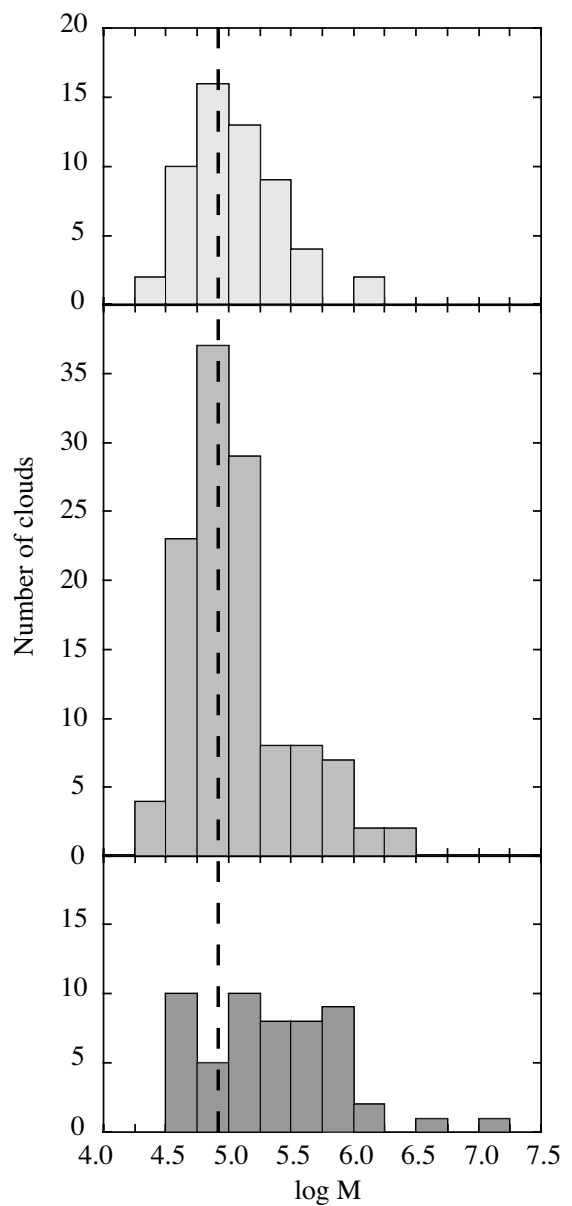

Figure 2. Mass distribution of Class I (Top), Class II (Middle), and Class III (Bottom) GMCs, respectively $\left(X=7 \times 10^{20} \mathrm{~cm}^{-2} \mathrm{~K}\right.$ $\mathrm{km} \mathrm{s}^{-1}$ is assumed; Fukui et al. 2006). sample covering the whole LMC enables us to infer the evolutionary timescales of the GMCs. We assume a steady state evolution and therefore the time spent in each phase is proportional to the number of the GMCs at each class. The absolute time scale is based on the age of young clusters, which is taken to be 10 Myr. The total lifetime of a GMC is then estimated to be $\sim 30$ Myr.

\section{Comparisons with supergiant shells}

A comparison of the GMCs and SGSs shows that clumpy GMCs are distributed along the edge of SGSs and only a few clouds are inside the SGSs except for the LMC 2. For simplicity, we assume the SGSs to be circles and introduce a "relative distance" between the GMCs and the SGSs, $d=D_{\mathrm{CO}, \mathrm{SGS}} / R_{\mathrm{SGS}}$ where $D_{\mathrm{CO}, \mathrm{SGS}}$ is the distance from the peak position of a GMC to the nearest SGS center, and $R_{\mathrm{SGS}}$ is the radius of the SGS (Fig. 3a). Here the GMC is just at the edge of the SGS if $d=1$.

Figure $3 \mathrm{~b}$ is a histogram of the relative distance with the expected frequency distribution obtained by distributing the same number of GMCs randomly in the observed area. By comparing the observed distribution with a random distribution, the number of GMCs is higher by a factor of $\sim 1.5$ near/at the edge of SGSs, $0.5<d<1.25$ and, particularly, by a factor of $\sim 2$ at the edge of $0.75<d<1.25$. We also found that the surface mass densities of the GMCs are

significantly enhanced around the edge of the SGSs by a factor of $\sim 2$. On the other hand, the number density of the clusters is enhanced by a factor of 4 inside the SGSs compared with the outer parts. The enhancement of the GMCs at the edge suggests the formation of GMCs due to an accumulation of the ISM and/or fragmentation of the SGSs (e.g., Ehlerová et al. 1997). The deficiency of GMCs in the central part suggests a dissipation of GMCs due to star-formation activities.

Next, we study where the clusters are formed in the GMC. We introduce here the position angle, $\theta$, as the angle between the two straight lines; one from a CO intensity peak to the center of the nearest SGS, and the other from the CO intensity peak to the associated cluster (Fig. 3a). If the position angle, $|\theta|<90^{\circ}$, the cluster is on the side facing the center of the SGSs. We study the position angles for the GMCs at the edge 

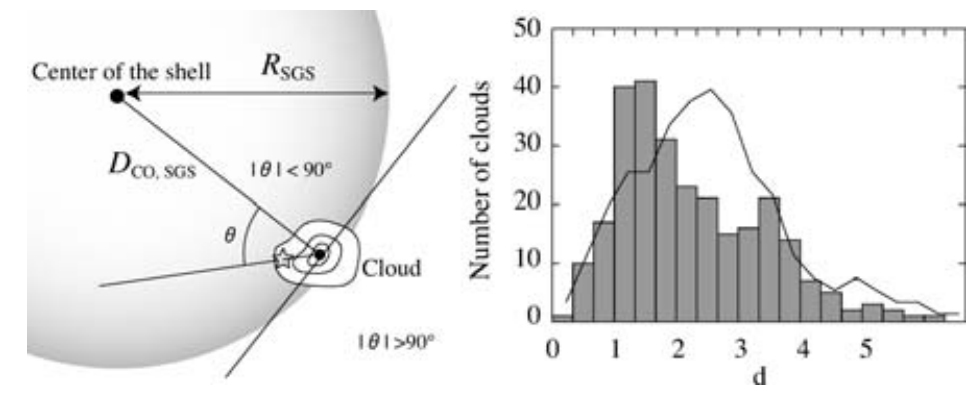

Figure 3. (a) Definition of the relative distance, $d$, and the position angle, $\theta$. (b) Histogram of the relative distances, $d$. The solid lines indicate the expected frequency distribution for the same number of samples randomly distributed in the observed area.

of the SGSs, $d=0.75-1.25$, and found that the number of clusters is by a factor of 2 greater in the GMCs facing to the center of the SGSs than other regions in the GMC.

These results are consistent with Yamaguchi et al. (2001a) and suggest that the SGSs play an important role in triggering the formation of molecular clouds and clusters.

\section{Acknowledgements}

The NANTEN project is based on a mutual agreement between Nagoya University and the Carnegie Institution of Washington. We greatly appreciate the hospitality of all the staff members of the Las Campanas Observatory. We are thankful to many Japanese public donors and companies who contributed to the realization of the project. We would like to acknowledge Drs. L. Staveley-Smith and M. Filipovic for the kind use of their radio continuum data prior to publication. This work is financially supported in part by a Grant-in-Aid for Scientific Research from the Ministry of Education, Culture, Sports, Science and Technology of Japan (No. 15071203) and from JSPS (No. 14102003, coreto-core program 17004 and No. 18684003).

\section{References}

Bica, E., Clariá, J. J., Dottori, H., Santos, J. F. C. Jr. \& Piatti, A. E. 1996, ApJS 102, 57

Brandl, B., Sams, B. J., Bertoldi, F., Eckart, A., Genzel, R., Drapatz, S., Hofmann, R., Loewe, M. \& Quirrenbach, A. 1996, APJ 466, 254

Davies, R. D., Elliott, K. H. \& Meaburn, J. 1976, MemRAS 81, 89

Dickel, J., Mclntyre, V. J., Guendl, R. A. \& Milne, D. K. 2005, AJ 129, 790

Filipovic, M. D., Jones, P. A., White, G. L. \& Haynes, R. F. 1998, AAS 130, 441

Ehlerová, S., Palouš, J., Theis, Ch. \& Hensler, G. 1997, A\&3A 328, 121

Fukui, Y., Kawamura, A., Minamidani, T., Mizuno, Y., Kanai, Y., Onishi, T., Mizuno, N., Yonekura, Y., Mizuno, A. \& Ogawa, 2006, ApJ submitted

Fukui, Y., Mizuno, N., Yamaguchi, R., Mizuno, A., Onishi, T., Ogawa, H., Yonekura, Y., Kawamura, A., et al. 1999, PASJ 51, 745

Kennicutt, R. C., Jr. \& Hodge, P. W. 1986, ApJ 306, 130

Kim, S., Dopita, M. A., Staveley-Smith, L. \& Bessell, M. S. 1999, AJ 118, 2797

Meaburn, J. 1980, MNRAS 192, 365

Rosolowsky, E. \& Leroy, A. 2006, PASP 118, 590

Tenorio-Tagle, G. \& Bodenheimer, P. 1988, ARAA 26, 145

Yamaguchi, R., Mizuno, N., Onishi, T., Mizuno, A. \& Fukui, Y. 2001a, PASJ 53, 959

Yamaguchi, R., Mizuno, N., Mizuno, A., Rubio, M., Abe, R.,Saito, H., Moriguchi, Y., Matsunaga, L., Onishi, T., Yonekura, Y. \& Fukui, Y. 2001b, PASJ 53, 985 


\section{Discussion}

Y.-H. CHU: The molecular cloud in the supergiant shell LMC-2 is associated with one of the two sheets of HI gas that confine the supergiant shell. To study the star formation in molecular clouds, YSOs identified by Spitzer observations are much better tracers of current star formation. We have seen star formation in molecular clouds that do not show any HII regions.

KAWAmURA: Yes. We shall present more detailed comparisons of GMCs and SGSs elsewhere. We see a very good correlation of HI and CO. In the present talk, we focussed on massive star formation, but obviously younger or low mass stars are better traced by new IR datasets as you suggest. We have started a comparison of Spitzer and Akari data with GMCs. These comparisons do show some indications of star formation of lower mass stars in the GMCs not associated with HII regions or clusters.

ZINNECKER: Can you comment on the lifetime of the giant molecular clouds in the LMC?

KAwAmURA: About $26 \%, 52 \%$, and $23 \%$ of the GMCs with $M>10^{5} \mathrm{M}_{\odot}$ are classified as class I, Class II and Class III. If the star formation proceed uniformly in time, and these classes represent an evolutionary sequence of the GMCs, we can also take this ratio as a ratio of the lifetime of the GMCs at each stage. The GMCs are thought to be dissipated about $5 \mathrm{My}$ after cluster formation, i.e., the life time of the Class III is about $5 \mathrm{My}$ because about a half of the young clusters with lifetimes less than $10 \mathrm{My}$ are associated with the GMCs. Taking these into account, the lifetime of the GMCs is considered to be about a few times $10 \mathrm{My}(20-30 \mathrm{My})$.

WALBORN: In your distinction between Class II and III molecular clouds, do you mean optically visible clusters in the latter? Presumably, the former have embedded or extincted clusters within/behind to power the HII regions. Comment: To refine these correlations, it will be important to discriminate young clusters by actual ages. Two, five, and ten Myr old clusters are very different with respect to stellar content and ISM interactions. Also, some massive clusters trigger second stellar generations around their peripheries, and some IR clusters may not be embedded but viewed through peripheral molecular clouds. (We could be very confused if we have a less fortunate line of sight toward 30 Doradus!)

KAWAMURA: We have compared the GMCs with HII regions seen in visible and/or radio and the optically visible young clusters and associations younger than $10 \mathrm{Myr}$. It is indeed important to discriminate young clusters according to more accurate age estimations as you point out. Nevertheless, we see a clear difference in mass between the GMCs with and without clusters of $<10$ Myrs. This suggests that the Class II and III are different groups of the GMCs. The current information of the age of the young clusters are limited, and it is not yet possible to have a sample of clusters with an accurate age estimation over the entire LMC. A comparison of the GMCs with clusters with more accurate age estimation will be carried out in the near future by using the recent IR studies by SAGE, Akari and SIRIUS. 